\title{
Peroxynitrite Modified Photoadducts as Possible Pathophysiological Biomarkers: A Short Review
}

\author{
Rizwan Ahmad ${ }^{1}$, Ashok Kumar Sah ${ }^{1}$ and Haseeb Ahsan ${ }^{2 *}$ \\ ${ }^{1}$ School of Life and Allied Health Sciences, Glocal University, Saharanpur, India \\ ${ }^{2}$ Faculty of Dentistry, Jamia Millia Islamia, Okhla, New Delhi, India
}

\begin{abstract}
Peroxynitrite, a toxic product of the free radicals, NO and superoxide, has been implicated in the pathologies of central nervous system, inflammatory diseases, including multiple sclerosis. Peroxynitrite leads to sulfhydryl oxidation and lipid peroxidation as well as structural and functional alteration of proteins. Endogenous peroxynitrite has been found to mediate various effects, including DNA single-strand breakage. Peroxynitrite is a relatively longlived oxidant that may serve as an important cytotoxic agent. Its biological effects are due to its reactivity toward a large number of molecules including lipids, amino acids and nucleic acids. It is involved in tissue damage in a number of pathophysiological conditions such as neurodegenerative diseases, cardiovascular disorders. A change in the structure of DNA could either be due to radiation or interaction with free radicals. Free radical species such as peroxynitrite make either native DNA or DNA-adducts immunogenic leading to the production of autoantibodies in autoimmune diseases such as systemic lupus erythematosus and rheumatoid arthritis.
\end{abstract}

Keywords: Peroxynitrite; DNA-adducts; DNA-arginine adduct; DNA-lysine adduct

\section{Introduction}

A DNA adduct is a piece of DNA covalently bonded to a chemical compound (carcinogenic, cancer causing). DNA adduct are used as biomarkers and as such are themselves measured to reflect the amount of cancer in the subjects, i.e. rats or other animals. Under experimental conditions, example of such DNA adducts is DMBA (7,12-dimethylbenz[a] anthracene). The DNA and protein adducts may act as a marker of genotoxicity and the determination of the interaction products (adducts) of a carcinogen indicates the amounts of genotoxic material that has reached tissues [1]. The crosslinking of genetic material to protein is one of the fundamental lesions produced in the biological system by sun ultraviolet light $[2,3]$. Lipid peroxidation products are also bound to DNA in the human liver and leukocytes. In chemical carcinogenesis, the formation of a carcinogen-DNA adducts is a critical step and therefore considered an important biomarker during the initiation stage. The inducibility of DNA adducts in vitro appear to be a risk factor in the development of some cancers. Cellular toxicity occurs when adduct formation disrupts the protein structure and function. Examples of DNA adducts are: cisplatin-DNA, polycyclic aromatic hydrocarbons-DNA, mitomycin C-DNA, anthramycin-DNA and DNA-lysine adducts [4]. Adduct formation results in increased secretion of messenger molecules, cytokines and chemokines that mediate communication among cells and promote inflammation e.g. tumor necrosis factor (TNF). Several neurodegenerative diseases such as Alzheimer's and Parkinson's as well as septic shock and inflammation involve formation of reactive oxygen and nitrogen species that include peroxynitrite (PON). Peroxynitrite can react with various endogenous biomolecules and antioxidants.

\section{Peroxynitrite}

Peroxynitrite (ONOO-), a reactive nitrogen species, is a potent oxidant and nitrating species formed from the reaction between nitric oxide (NO) and superoxide radicals $\left(\mathrm{O}_{2}^{-}\right)$. Free radicals like peroxynitrite create oxidative stress which may damage biological macromolecules and causes malfunctioning of cellular functions. Peroxynitrite is produced by the body in response to a variety of toxicologically relevant molecules including environmental toxins. It is also produced by the body in reperfusion injury and inflammation. Peroxynitrite is a potent trigger of DNA strand breakage and activates nuclear enzyme poly[ADP-ribose] polymerase (PARP) resulting in energy depletion and necrosis of the cells. The attenuation of peroxynitrite is of significant therapeutic benefit from environmental toxins as well as in a variety of inflammatory and reperfusion disease conditions [5].

\section{Effect of peroxynitrite on DNA-protein adducts}

Peroxynitrite is an extremely short lived species. It reacts with tyrosyl residues to form 3-nitrotyrosine. Peroxynitrite may also lead to necrosis and reactive nitrogen species have also been appreciated as signaling molecules [6]. Nitric oxide cause deleterious effects in various human pathologies such as neurological disorders, artherosclerosis and ischemia reperfusion [7]. Exposure of amino acids, peptides and proteins to gamma radiation and peroxynitrite in presence of oxygen, gives rise to hydrogen peroxide. Hydrogen peroxide damages nuclear proteins resulting in oxidative damage to associated DNA. These hydrogen peroxide derived radicals reacts with pyrimidine DNA bases and nucleosides leading to formation of adducts causing transversion mutations [8].

Effect of peroxynitrite on DNA-arginine and DNA-lysine adducts

Adducts arises from the chemical modification of bases in DNA

${ }^{*}$ Corresponding author: Dr. Haseeb Ahsan, Department of Biochemistry, Faculty of Dentistry, Jamia Millia Islamia, Okhla, New Delhi-110015, India, E-mail: drhahsan@gmail.com

Received October 28, 2015; Accepted November 23, 2015; Published November 26, 2015

Citation: Ahmad R, Sah AK, Ahsan H (2015) Peroxynitrite Modified Photoadducts as Possible Pathophysiological Biomarkers: A Short Review. J Mol Biomark Diagn 6: 263. doi:10.4172/2155-9929.1000263

Copyright: @ 2015 Ahmad R, et al. This is an open-access article distributed under the terms of the Creative Commons Attribution License, which permits unrestricted use, distribution, and reproduction in any medium, provided the original author and source are credited. 
or amino acids in protein by toxic chemicals and high energy UV radiation. Arginine is an amino acid that has potential to form DNAprotein photocrosslinks. Determination of the interaction product (adduct) of a carcinogen with DNA or protein indicates the amount of genotoxicity $[9,10]$. DNA adducts give further information to the DNA damage levels as low as one adducts per $10^{8}$ nucleotides can be measured [11]. Cellular toxicity occurs when adduct formation disrupts protein structure and function, which secondarily cause damage to submembrane organelles, metabolic pathways, or cytological processes. Neurotoxicants can form adducts with nucleophilic residues on proteins. Proteins adducts play a causal role in generation of neurotoxicity and adduct formation also leads to neurotoxicity [12].

We have studied the photochemical addition of arginine to native DNA. The UV spectroscopic analysis of the DNA-arginine photoadduct showed hyperchromicity, indicating the formation of single stranded breaks in DNA due to photomodification. It may be attributed to the destabilization of the double helix due to the singlestranded regions in DNA-arginine adduct as a result of peroxynitrite modification. Adducts also induce immune responses and antibodies have been developed against many carcinogen-DNA adducts as well as UV damaged and oxidized bases [13]. Lysine and arginine-rich histones in nucleosomes on modification by environmental agents form histone-DNA adducts, making it immunogenic. Alteration of DNA resulting from photomodification or peroxynitrite could lead to the development of antibodies or mutations to modified DNA [14-17].

A change in the structure of DNA could either be due to radiation or due to damage with free radicals [18]. Since there are many polybasic compounds in the vicinity of DNA, there exists a possibility of their interaction with DNA on exposure to radiation or free radicals. Lysine and arginine-rich histones in nucleosomes on modification by environmental agents form histone-DNA adducts, making it immunogenic. It appears that the pathogenic anti-DNA autoantibodies are generated through some modified epitopes on nucleic acids [18]. Prominent DNA modifications induced by exposure to peroxynitrite include the formation of 8-nitro-guanine and 8-oxyguanine as well single-strand breaks [19]. Peroxynitrite reacts significantly with guanine, which upon oxidation and nitration leads to mutagenicity and strand breaks, respectively. Peroxynitrite also damages DNA by covalent bond formation and removal of DNA bases $[20,21]$.

Similarly, the UV spectroscopic analysis of the DNA-lysine photoadduct showed hyperchromism, indicating either the formation of single-stranded breaks in DNA or helix destabilization at the site of lysine conjugation. Peroxynitrite causes substantial damage to the DNA-lysine adduct as evident from the hyperchromicity of the spectral curve, which could be attributed to strand breaks. On peroxynitrite modification, the hypochromicity increased, which may be due to the shielding effect of lysine or it may also be attributed to the extensive cross-linking between peroxynitrite and the DNA-lysine adduct [21]

\section{Conclusion}

Peroxynitrite (ONOO-), a toxic product of the free radicals, NO and superoxide, has been implicated in the pathologies of inflammatory diseases, including multiple sclerosis. Peroxynitrite leads to sulfhydryl oxidation and lipid peroxidation as well as structural and functional alteration of proteins and DNA. Peroxynitrite participates in neuron damage in association with inflammatory processes. Endogenous peroxynitrite has been found to mediate various effects, including DNA single-strand breakage. In addition to beneficial effects in host defense mechanism, the anion may have deleterious effects on host tissues. The relative balance between these radicals has important implications for vascular pathophysiology in hypertension and other vascular conditions. A change in the structure of DNA could either be due to radiation or interaction with free radicals. Peroxynitrite radical make native proteins and DNA immunogenic. The modified antigens leads to production of anti-DNA auto antibodies in autoimmune disorders such as systemic lupus erythematosus. Hence, these modified biomolecules and photoadducts could have important implications in several pathophysiological conditions [22].

\section{Acknowledgment}

RA, AKS are grateful to the management of Glocal University, Saharanpur, India for the support during the preparation of this manuscript.

\section{Conflict of Interest}

The authors declare that there is no conflict of interest.

\section{References}

1. Phillips DH, Venitt $S$ (2012) DNA and protein adducts in human tissues resulting from exposure to tobacco smoke. Int J Cancer 131: 2733-2753.

2. Wani A, Mushtaq S, Ahsan H, Ahmad R (2013) Characterization of human glycated DNA modified with peroxynitrite. Helix 1: 221-225.

3. Menck CF, Munford V (2014) DNA repair diseases: What do they tell us about cancer and aging? Genet Mol Biol 37: 220-233.

4. Boffetta P, Islami F (2013) The contribution of molecular epidemiology to the identification of human carcinogens: current status and future perspectives. Ann Oncol 24: 901-908.

5. Koppenol WH, Kissner R, Beckman JS (1996) Syntheses of peroxynitrite: to go with the flow or on solid grounds? Methods Enzymol 269: 296-302.

6. Zuckerbraun BS, George P, Gladwin MT (2011) Nitrite in pulmonary arteria hypertension: therapeutic avenues in the setting of dysregulated arginine/nitric oxide synthase signalling. Cardiovasc Res. 89: 542-52.

7. Sharma RA, Farmer PB (2004) Biological relevance of adduct detection to the chemoprevention of cancer. Clin Cancer Res 10: 4901-4912.

8. Farmer PB (2004) DNA and protein adducts as markers of genotoxicity. Toxicol Lett 149: 3-9.

9. Farmer PB, Singh R (2008) Use of DNA adducts to identify human health risk from exposure to hazardous environmental pollutants: the increasing role of mass spectrometry in assessing biologically effective doses of genotoxic carcinogens. Mutat Res 659: 68-76.

10. Farmer PB, Brown K, Tompkins E, Emms VL, Jones DJ, et al. (2005) DNA adducts: mass spectrometry methods and future prospects. Toxicol Appl Pharmacol 207: 293-301.

11. Vinten-Johansen J (2000) Physiological effects of peroxynitrite: potentia products of the environment. Circ Res 87: 170-172.

12. Ohshima H, Bartsch $\mathrm{H}$ (1994) Chronic infections and inflammatory processes as cancer risk factors: possible role of nitric oxide in carcinogenesis. Mutat Res 305: 253-264.

13. Rabinovitch A (1992) Free radicals as mediators of pancreatic islet beta-cell injury in autoimmune diabetes. J Lab Clin Med 119: 455-456.

14. Dedon PC, Tannenbaum SR (2004) Reactive nitrogen species in the chemica biology of inflammation. Arch Biochem Biophys 423: 12-22.

15. Grune T, Blasig IE, Sitte N, Roloff B, Haseloff R, et al. (1998) Peroxynitrite increases the degradation of aconitase and other cellular proteins by proteasome. J Biol Chem 273: 10857-10862.

16. Thomas DD, Ridnour LA, Isenberg JS, Flores-Santana W, Switzer $\mathrm{CH}$, et al (2008) The chemical biology of nitric oxide: implications in cellular signaling Free Radic Biol Med 45: 18-31.

17. Nair J, Barbin A, Velic I, Bartsch H (1999) Etheno DNA-base adducts from endogenous reactive species. Mutat Res 424: 59-69.

18. Ohshima H, Yoshie Y, Auriol S, Gilibert I (1998) Antioxidant and pro-oxidan actions of flavonoids: effects on DNA damage induced by nitric oxide, peroxynitrite and nitroxyl anion. Free Radic Biol Med 25: 1057-1065. 
Citation: Ahmad R, Sah AK, Ahsan H (2015) Peroxynitrite Modified Photoadducts as Possible Pathophysiological Biomarkers: A Short Review. J Mol Biomark Diagn 7: 263. doi:10.4172/2155-9929.1000263

19. Szabó C, Ischiropoulos H, Radi R (2007) Peroxynitrite: biochemistry, pathophysiology and development of therapeutics.Nat Rev Drug Discov 6: $662-680$

20. Yermilov V, Rubio J, Becchi M, Friesen MD, Pignatelli B, et al. (1995) Formation of 8 -nitroguanine by the reaction of guanine with peroxynitrite in vitro. Carcinogenesis 16: 2045-2050.
21. Ahmad R, Rasheed Z, Kaushal E, Singh D, Ahsan H (2008) Biochemical Evaluation of Human DNA-Lysine Photoadduct Treated with Peroxynitrite. Toxicol Mech Methods 18: 589-595.

22. Ahmad R, Ahsan H (2014) Role of peroxynitrite-modified biomolecules in the etiopathogenesis of systemic lupus erythematosus. Clin Exp Med 14: 1-11. 